\title{
In-flight calibration of STEREO-B/WAVES antenna system
}

\author{
M. Panchenko, ${ }^{1}$ W. Macher,${ }^{1}$ H.O. Rucker,${ }^{1}$ G. Fischer, ${ }^{1}$ T. H. Oswald, ${ }^{1}$ B. \\ Cecconi, ${ }^{2}$ M. Maksimovic, ${ }^{2}$
}

The STEREO/WAVES experiment (SWAVES) onboard the two STEREO spacecraft (Solar TErrestrial RElations Observatory) launched on Oct 25, 2006, is dedicated to the measurement of the radio spectrum at frequencies between a few $\mathrm{kHz}$ and $16 \mathrm{MHz}$. The SWAVES antenna system consists of $6 \mathrm{~m}$ long orthogonal monopoles designed to measure the electric component of the radio waves. With this configuration direction finding of radio sources and polarimetry (analysis of the polarization state) of incident radio waves is possible. For the evaluation of the SWAVES data the receiving properties of the antennas, distorted by the radiation coupling with the spacecraft body and other onboard devices, have to be known accurately. In the present context, these properties are described by the antenna effective length vectors. We present the results of an in-flight calibration of the SWAVES antennas using the observations of the non-thermal terrestrial Auroral Kilometric Radiation (AKR) during STEREO roll maneuvers in an early stage of the mission. A least squares method combined with a genetic algorithm was applied to find the effective length vectors of the STEREO-B/WAVES antennas in a quasi-static frequency range ( $L_{\text {antenna }} \ll \lambda_{\text {wave }}$ ) which fit best to the model and observed AKR intensity profiles. The obtained results confirm the former SWAVES antenna analysis by rheometry and numerical simulations. A final set of antenna parameters are recommended as a basis for evaluations of the SWAVES data.

\section{Introduction}

The complex radiation coupling between the antennas aboard a spacecraft and other electric devices or metallic structures alters currents induced in the electric antennas by an incident electromagnetic wave, and, therefore, causes the distortion of the reception properties. As result the effective length vectors, which describe the main antenna reception properties such as directional characteristics and effective length, differ from the expected ones based on properties of the "stand-alone" antennas. Therefore, for the accurate evaluation of the data acquired by the radio instruments onboard spacecraft the reception properties of the antennas, influenced by the spacecraft body, have to be known precisely enough.

\footnotetext{
${ }^{1}$ Space Research Institute, Austrian Academy of Sciences, Schmiedlstrasse 6, A-8042 Graz, Austria

${ }^{2}$ LESIA, Observatoire de Paris, 5, place Jules Janssen, 92195 MEUDON Cedex, France.
}

Copyright 2021 by the American Geophysical Union. 0048-6604/21/\$11.00
This can be done by means of several well developed techniques [Macher, 2008], such as rheometry [Rucker et al., 1996; Macher et al., 2007], anechoic chamber measurements [Riddle, 1976], in-flight calibration [Vogl et al., 2004; Panchenko, 2004; Cecconi and Zarka, 2005] or computer simulations [Oswald et al., 2009; Rucker et al., 2011].

The rheometry method uses the scaled model of the spacecraft suspended in an electrolytic tank and the antenna reception properties are determined by measuring a response of the electric antennas to the electric field in the quasi-static frequency range, i.e. when the radio wavelength is much greater than the antenna length (short electric antenna) [Rucker et al., 1996; Macher and Oswald, 2011]. A very powerful technique to analyze the reception properties of the spaceborne antennas is a computer-based wire or patch-grid simulation in which the spacecraft body and antennas are modeled as mesh of wires or patches [Fischer et al., 2001; Oswald et al., 2009; Sampl et al., 2011, 2012]. Numerical solutions of the electric and magnetic field integral equations by means of numerical electromagnetic codes yield the satisfying determination of the antenna effective length vectors and 
antenna radiation pattern for quasi-static as well as for higher frequency range. These computer simulations allow to predict the reception properties of the antennas before launch of the spacecraft.

The main idea of the in-flight calibration is the determination of the reception properties of the antennas after spacecraft launch using the natural radio sources such as galactic background or planetary radio emission. An in-flight calibration procedure consist of two main parts: 1) determination of the antenna system gain including effective lengths of the antennas as well as base and antenna capacitances, and 2) determination of the antenna directivity - i.e. the directions of the antenna effective lengths vectors.

The antenna gain and effective lengths can be determined using the quasi-isotropic non-thermal galactic background as a reference radio source. This method has been implemented to derive the absolute flux density measurements of the Cassini/RPWS [Zarka et al., 2004] and the effective length of the STEREO/WAVES electrical antenna system $[Z a-$ slavsky et al., 2011].

The effective antenna length vectors of the electric antennas can be investigated by analyzing the temporal variation of the intensity of the radio emission emitted from the point source with known location. In this method the spaceborne antenna system must rotate with respect to the direction of the incident radiation. The in-flight calibration method is one of the most reliable techniques to determine the reception properties of the antenna because the method deals with the real observations. The methodology of this calibration is well described in Vogl et al. [2004]. The method has successfully been applied to derive the antenna properties of radio experiments onboard e.g. ISEE-3 [Fainberg et al., 1985], Voyager [Lecacheux and Ortega-Molina, 1987; Wang and Carr, 1994], Interball-2/Polrad [Panchenko, 2004] or Cassini [Vogl et al., 2004; Cecconi and Zarka, 2005]

In this paper we present the results of determining the effective length vectors of the antennas of the WAVES instrument onboard STEREO spacecraft. We used the terrestrial Auroral Kilometric Radiation (AKR) - intense radio emission from auroral regions [Gurnett, 1974], as a radio source. The antenna effective length vectors have been determined by fitting of the model-predicted temporal variations to the time profiles of the AKR intensity measured during STEREO/WAVES roll maneuvers. The ob- tained results are compared with antenna effective length vectors evaluated by methods of rheometry and numerical computer simulation.

The STEREO/WAVES radio experiment is described in chapter 2. The method of in-flight calibration of the SWAVES antenna is presented in section 3 , and the results and error analysis are given in section 4 . In section 5 we discuss the results.

\section{STEREO/WAVES antenna system}

Solar TErrestrial RElations Observatory (STEREO) consists of two identical spacecraft (STEREO-A and STEREO-B), launched on October 25, 2006. The main goals of the STEREO mission are investigations of the three-dimensional structure and evolution of the solar coronal mass ejections (CMEs) as well as the study of the CMEs interaction with the Earth's magnetosphere. The set of scientific experiments onboard STEREO provide complex measurements of the electromagnetic and local plasma waves, as well as 3D magnetic field components, and plasma parameters like the solar wind bulk velocity, density and temperature. After several highly eccentric geocentrical orbits and lunar swing-by maneuvers (January 2007) the two STEREO spacecraft split up and were inserted into a heliocentric orbit. One of the spacecraft, STEREO-A (Stereo Ahead) leads Earth in an orbit which is slightly closer to the Sun, while the other spacecraft, STEREO-B (STEREO Behind) trails Earth orbiting slightly outside the terrestrial orbit.

The SWAVES radio experiment is an interplanetary radio burst tracker that observes the generation and propagation of the radio disturbances from the Sun to the orbit of Earth [Bougeret et al., 2008]. SWAVES consists of fixed, high (HFR) and low (LFR) frequency receivers as well as of a time domain sampler (TDS). The antenna system of the SWAVES instrument include three nearly mutually orthogonal electric monopoles $X, Y$ and $Z$. Each of the monopoles has a length of 6 meters.

The super-heterodyne swept frequency receiver HFR (High Frequency Receiver) includes two separate parts (or "logical receivers") HFR1 and HFR2 which cover the frequency ranges $0.125-2 \mathrm{MHz}$ and 2-16 MHz, respectively, with $50 \mathrm{kHz}$ frequency resolution. The time resolution depends on the working mode. HFR1 can work in direction finding mode providing spectral and complex cross-spectral 
power densities of incident waves. HFR1 is a dual channel receiver which can be connected to a pair of monopoles $X, Y$ or $Z$ or to combinations of monopoles and "pseudo dipoles" (monopoles $X$ or $Z$ can be paired with the $Y$ antenna). Thanks to the two parallel analysis channels, HFR1 measures instantaneously four values: autocorrelation of each of the two antennas and the real and imaginary part of cross-correlation between pairs of the antennas (see Bougeret et al. [2008] for more details).

In the so-called direction finding mode (DF) the HFR 1 switches between the pairs of the antenna at each frequency step, providing quasi-instantaneous auto and complex cross-spectra density of the wave between different antenna combination. HFR1 is operated in two special direction findings modes:

DF1 - "two antenna mode", in which two possible antenna configurations are used:

DF1 '13' - one monopole $E_{X}$ and one dipole $E_{Z Y}$ (dipole consist of $E_{Z}$ and $E_{Y}$ monopoles). The output signals in this mode are: auto correlations $\left(A_{X}, A_{Z Y}\right)$ and cross-correlations $\left(\Re_{X / Z Y}\right)$ and $\left(\Im_{X / Z Y}\right)$.

DF1 '31' - one monopole $E_{Z}$ and one dipole $E_{X Y}$ (monopoles $E_{X}$ and $E_{Y}$ are connected as a dipole). Operating in this mode HFR1 at each frequency provides four quasi-instantaneous output signals: two auto correlations $\left(A_{Z}, A_{X Y}\right)$ and the real $\left(\Re_{Z / X Y}\right)$ and the imaginary $\left(\Im_{Z / X Y}\right)$ part of the cross-correlation between $E_{Z}$ and $E_{X Y}$.

DF2 - "three antenna mode", uses sequence combination of all monopoles $E_{X}, E_{Y}, E_{Z}$. This mode yields nine quasi-instantaneous output signals: three auto correlations $\left(A_{X}, A_{Y}, A_{Z}\right)$; three real parts $\left(\Re_{X Y}, \Re_{Y Z}, \Re_{Z X}\right)$ and three imaginary parts $\left(\Im_{X Y}, \Im_{Y Z}, \Im_{Z X}\right)$ of cross-correlations.

\section{Method and data preparation}

\subsection{AKR observation}

Several spacecraft roll maneuvers have been scheduled at the beginning of the mission. During this maneuvers the spacecraft were at $90-140 R_{E}$ away from the Earth. Each maneuver lasted 10 hours and consisted of 10 consecutive rolls $\left(6^{\circ}\right.$ per minute). In particular, STEREO-A performed three roll maneuvers: 1st on 12/18/2006 06:50-16:50, 2nd on 12/20/2006 07:20-17:20 and 3rd on 12/23/2006 04:30-14:30. Second spacecraft, STEREO-B, performed only two roll maneuvers on 01/29/2007 05:10-

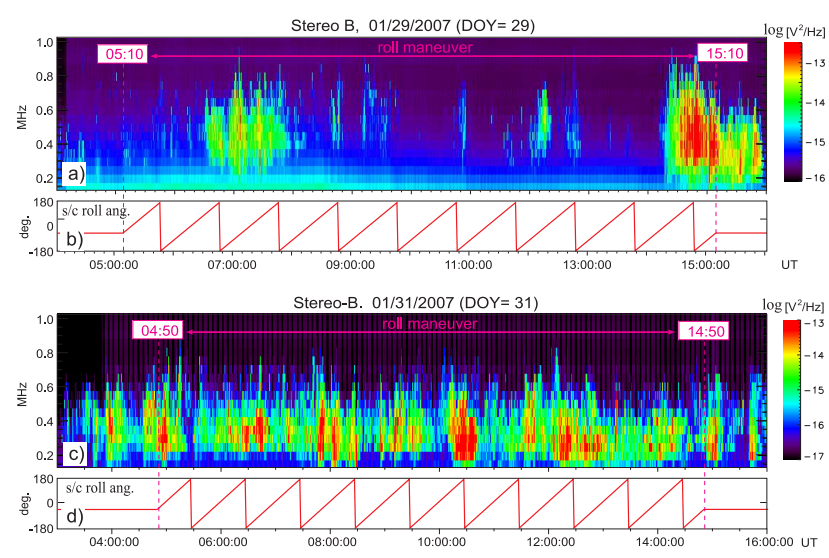

Figure 1. Dynamic spectra of the radio emission recorded by STEREO-B on 29 and 31 Jan., 2007 (panels a and c). Terrestrial AKR is observed in a frequency range up to $700-800 \mathrm{kHz}$. The panels b) and d) show the roll angle of the spacecraft in the GSE coordinate frame. This angle shows the times when STEREO-B performed roll maneuvers: on 29/01/2007 between 05:10-15:10 UT and 31/01/2007 between 04:50-14:50 UT.

15:10 and 01/31/2007 04:50-14:50. These spacecraft roll maneuvers can be used to determinate the effective length vector of each antenna.

The in-flight calibration requires the observation of the radio emissions from a known position relative to the rotating antenna system. During the roll maneuvers only one spacecraft, STEREO-B, observed the terrestrial Auroral Kilometric Radiation (AKR) with the signal/noise ratio sufficiently high to perform the antenna calibration. The reason why only STEREO-B observed AKR is that, after the last lunar swing-by maneuver, STEREO-B flew by the Earth crossing the dusk side of the magnetosphere (from dayside to nightside). Such a trajectory enabled a long-lasting (several months) observation of the terrestrial AKR (see [Panchenko et al., 2009]), whose sources are known to be merely fixed in local magnetic time of the dusk-night part of the magnetosphere. In the same time STEREO-A was on the dayside magnetic local time sector and, therefore, it was not able to observe the AKR.

Fig. 1 shows the dynamic radio spectra recorded by STEREO-B during SWAVES antenna rolls on 29 and 31 Jan., 2007. The bottom panels (b) and (d) show the roll angle of the spacecraft in the GSE (Geocentric Solar Ecliptic) coordinate frame. The roll angle as well as the spacecraft 
attitude and position have been calculated using the NASA NAIF/SPICE toolkit - a system which provides spacecraft ancillary information (http : //naif.jpl.nasa.gov/naif/toolkit.html).

As shown in Figure 1 only in one episode, i.e. on 31 Jan. 2007, AKR was observed continuously during the whole roll maneuver, whereas on 29 Jan. 2007 the radio emission was detected only during two short time spans, i.e. 06:30-08:00 and 14:15-15:20. Therefore we have decided to use only the observations performed on 31 Jan., 2007 between 04:50 and 14:50 UT. During these time intervals HFR1 was operated consecutively in DF1 '13', DF1 ' 31 ' and DF2 modes - i.e. in each consecutive frequency sweep HFR1 was switched between DF1 '13', DF1 '31' and DF2 modes.

The first step of the data preparation deals with a subtraction of the frequency dependent background which consists of receiver and galactic background noises. The level of the background has been determined in the same way as it was done for Cassini/RPWS calibration [Vogl et al., 2004, appendix A ]. In particular, assuming that the background is nearly constant with time and depends only on the frequency and working mode of the receiver the background level was determined using a histogram of occurrence probabilities of intensities calculated for each frequency. The histograms were obtained during time intervals when no AKR activity was detected. Then, the background level at each frequency was defined as the intensity which corresponds to the lower $5 \%$ occurrence level (left from the maximum of the occurrence histogram) (see Zarka et al. [2004]). The data points below the background were excluded from the analysis in order to avoid any bias in our data set. Since STEREO-B/WAVES was operated mainly in DF2 mode (in the beginning of the mission ) the frequency dependent background was calculated only for this mode. The background for the DF1 mode has been assumed to be at the same level as for DF2 mode, although, as was shown by Taubenschuss [2005] the background can be a slightly different for different antenna combination. It is worth to note, that the intensity of the AKR observed on 31 Jan. 2007 was $2-3$ orders of magnitude higher than the averaged background level at frequencies below $1 \mathrm{MHz}$. Therefore, any potential inaccuracies in background subtraction do not play a significant role in the antenna calibration procedure.
After the background subtraction, the intensity profiles have been established by an averaging of the data over the frequency bandwidth $225-475 \mathrm{kHz}$ (6 frequency channels of the HFR1 receiver). These normalized intensity profiles are plotted as solid blue lines in Figure 3. The standard deviation of each mean value will be used as uncertainties of the observations $\sigma_{i}$ in equation (3).

\subsection{Model predicted SWAVES outputs}

The open-circuit voltages on each short electrical dipole (or input voltages at each receiver channel) can be expressed as:

$$
V_{i}=\overrightarrow{h_{i}} \vec{E}
$$

where $\vec{h}_{i}$ is an effective antenna length vector, $\vec{E}$ is the electric wave vector of the incoming wave and $i$ denotes the antenna, i.e. $i=X, Y, Z$ for DF2 mode or $i=X Y, Z$ and $i=X, Z Y$ for DF1 mode. Each $\vec{h}$ vector is represented by the following spherical coordinates: the antenna length $h_{i}$ and the colatitude $\zeta_{i}$ and the azimuth $\xi_{i}$ angles. We chose the same coordinate system, as was used by Oswald et al. [2009] for rheometry and wire-grid calibrations of the SWAVES antenna. In particular, the colatitudes $\zeta_{x, y, z}$ of each SWAVES effective antenna vectors as well as the colatitude of the AKR source $\zeta_{s}$ are defined as an angle from the spacecraft axis $X$ towards $Y_{s c} Z_{s c}$ plane. The azimuth angles $\xi_{X, Y, Z}$ and $\xi_{s}$ are counted from $-Z_{s c}$ towards $Y_{s c}$.

The sample covariance of the $V_{i}$ in equation (1) which are actually measured by the receiver is linearly related to the covariance of the incoming wave electric field (which basically forms the source polarization vector). Then, in the spacecraft coordinates the model-predicted analytic signals of the output voltages at each receiver can be written as [Ladreiter et al., 1995; Vogl et al., 2004; Cecconi and Zarka, 2005]:

$$
\begin{aligned}
\Re\left(\left\langle V_{i} V_{j}^{*}\right\rangle\right)= & 0.5 S h_{i} h_{j}\left[(1+Q) \Omega_{i} \Omega_{j}+\right. \\
& \left.U\left(\Omega_{i} \Psi_{j}+\Omega_{j} \Psi_{i}\right)+(1-Q) \Psi_{i} \Psi_{j}\right] \\
\Im\left(\left\langle V_{i} V_{j}^{*}\right\rangle\right)= & 0.5 S h_{i} h_{j} V\left[\Omega_{i} \Psi_{j}-\Omega_{j} \Psi_{i}\right]
\end{aligned}
$$

where $\Omega_{i, j}=\cos \zeta_{i, j} \sin \zeta_{s}-\sin \zeta_{i, j} \cos \zeta_{s} \cos \left(\phi_{s}-\right.$ $\left.\phi_{i, j}\right), \Psi_{i, j}=-\sin \zeta_{i, j} \sin \left(\xi_{s}-\xi_{i, j}\right), \zeta_{s}$ and $\xi_{s}$ colatitude and azimuth of the radio source in the spacecraft coordinates; $V_{i, j}$ - analytical signals of the input voltages at each receiver; $h_{i, j}, \zeta_{i, j}, \xi_{i, j}$ - effective 
length, colatitude and azimuth of electric antennas; $S, Q, U, V$ - Stokes parameters of the incoming wave, asterisk denote complex conjugate and $\langle\ldots\rangle$ is a timeaveraging operation.

During the spacecraft roll maneuvers SWAVES/ HFR1 was sequentially switched between DF1 '13', DF1 '31' and DF2 modes for each measurement sweep. Therefore, the model-predicted signals are expressed the system of 4 equations in case of the 2 antenna modes, DF1 ' 13 ' and DF1 ' 31 ' or 9 equations for DF2 mode. The coefficients of these equations contain non-linear expressions of parameters describing the the source direction (in case of point source approximation), Stokes parameters of the wave and the components of the antenna effective length vectors (colatitude, azimuth and length).

With a distant observation at $\approx 110 R_{E}$ away from Earth the AKR radio sources can be assumed as a point source. During the roll maneuvers on 31 Jan., 2007 STEREO-B was at high latitudes over the Northern hemisphere and therefore was able to observe only the Northern AKR sources which are mainly located in the evening sector of the magnetosphere i.e. between $\sim 18: 00-22: 00$ hours of the magnetic local time (MLT) and $\sim 65-75^{\circ}$ of the invariant magnetic latitude(Hanasz et al. [2003]; Mutel et al. [2004]). In our calculations we assumed that the AKR source is located at 21:00 of MLT, $70^{\circ}$ of invariant magnetic latitude and at the altitude of $0.8 R_{E}$ above the Earth surface (corresponds to the sources at $\sim 300 \mathrm{kHz}$.) Therefore, the radio source coordinates in equation (2) are assumed as known parameters. Moreover, using the well known AKR polarization characteristic, i.e. that AKR is fully circularly polarized (e.g. Panchenko et al. [2008]) with domination of the right-handed R-X mode (Hanasz et al. [2003]) as well as assuming that the polarization state of the AKR does not change during the observation, we can suggest that the Stokes parameters are $Q=U=0$ and $V=-1$. In addition, by assuming a perfect subtraction of the background contribution, one can see that the linear system of equations (2) is homogeneous and therefore it can be normalized to the unknown source intensity parameter, and only the antenna effective length ratio retained.

Due to the errors in the measurements we cannot expect an exact solution of the equations (2). Therefore the best solution in the least square sense is sought, i.e. the sum of the squares of the differences between the model predicted values $\left(P^{\text {mod }}\right)$ and the observations $\left(P^{o b s}\right)$ is minimized:

$\chi^{2}=\sum_{m=1}^{M} \sum_{n=1}^{N} \sigma_{m, n}^{-2}\left(\frac{P_{m, n}^{o b s}\left(t_{n}\right)}{I_{n}^{\text {obs }}}-\frac{P_{m, n}^{\bmod }(X)}{I_{n}^{\bmod }}\right)^{2}=\operatorname{min(23)}$

where $\mathbf{X}=\left\{\zeta_{s}, \xi_{s}, h_{i, j}, \zeta_{i, j}, \xi_{i, j}, S, Q, U, V\right\}$ are the input parameters of the model described by equations (2)), index $m$ counts the output receiver channels (e.g. for DF2 mode: $m=1$ denotes $A_{X}$, $m=2$ corresponds to $A_{Y}$, etc.), $M$ is the total number of the receiver output channels, index $n$ denotes each data point, $N$ is the total number of data points in given time series and $\sigma_{n, m}$ represents uncertainties of each observation $P_{n, m}^{o b s}$. The normalization coefficients in equation(3) are : $I_{n}^{o b s, \bmod }=P_{X, n}^{o b s, \bmod }+P_{Y, n}^{o b s, \bmod }+P_{Z, n}^{o b s, \bmod }$ for DF2 mode, $I_{n}^{o b s, \text { mod }}=P_{X, n}^{o b s, \text { mod }}+P_{Z Y, n}^{o b s, \text { mod }}$ for DF1 ' 13 ' mode and $I_{n}^{\text {obs, mod }}=P_{Z, n}^{\text {obs, mod }}+P_{X Y, n}^{\text {obs, } \bmod }$ for DF1 '31'.

Equation (3) can be used only in assumption that the errors of observations are uncorrelated. This implies that the variance-covariance matrix of observations whose elements $\sigma_{m, n}$ are weights for the squared residuals is diagonal. In fact, when the antenna system receives the strongly polarized radio signal (e.g. AKR) one can expect cross correlation between uncertainties of the measurements and as a result the non-zero off-diagonal elements in the variance-covariance matrix. Therefore, in more general case methods of the variance-covariance matrix estimation described in Lecacheux [2011] should be used. In our calculations we used the observations with large signal/noise ratio and the expected deviations of the electric antenna vectors from orthogonality are not large. Therefore we assumed that the off-diagonal elements in variance-covariance matrix are very small and we can use the equation (3).

The non-linear problem (3) can be solved by means of non-linear optimization methods. Most of these methods are based on iterative procedures: from a starting point $x_{0}$ the method produces a series of vectors $x_{1}, x_{2}, \ldots x_{n}$ which converge to $x_{\text {true }}$ - the local minimizer for the function $\chi^{2}(x)$. The main problem of such an algorithm is that the system of equations can be ill-conditioned (numerically very close to singularity) in certain regions of the parameters space, with no unique solution. This is a well-known problem when the inversion is applied. In general, the ill-conditioned system of equations describes the situation when small fluctuations of the input data may result in a drastic increase of errors in the solu- 
tion. The other problem of the nonlinear optimization method is the definition of the initial guess of the model parameters. Unfortunate choosing of the initial guess can result in finding local minima of $\chi^{2}(x)$ which are much larger than its global minimum.

\subsection{Genetic algorithm (GA)}

Recently the stochastic global search techniques, such as genetic algorithms, became a powerful tool to solve inverse problems. The genetic algorithm (GA), the concept of which has been introduced by Holland [1975], is a stochastic technique based on the biological principle of survival of the fittest. The method represents each potential solution of the optimization problem as a set of chromosomes (genome). The algorithm begins from initialization of the initial population of randomly selected "individuals" (potential solutions). Each "individual" contains a set of chromosomes which represent the free parameters of the optimization problem. Applying the genetic operators such as mutation and crossover the fittest "individuals" (potential solutions with the best approximation of the problem) are determined on each iteration step. These fittest "individuals" have a greater chance to leave most offspring forming a next population to which the mutation and crossover operators are applied again. In this way, a successive approximation of the solution of the optimization problem is achieved improving gradually. The application of the GA in astronomy and astrophysics is well reviewed by Charbonneau [1995].

The main advantage of the GA is that this algorithm doesn't require a good initial guess of the solution and therefore can operate in the whole space of the free parameters of the model. Therefore, unlike "classical" nonlinear optimization methods, the GA is significantly less sensitive to misleading local minima and in most cases the global extremum of the function can be found. Genetic algorithms can also be adopted to solve ill-conditioned inverse problems [e.g. Mera et al., 2004]. These advantages are countered by fact that the GA requires higher computing power. Moreover, since GA is a stochastic method which uses random search, the resulting extremum of the optimization task is an extremum only in probabilistic sense. In other words, the obtained results with some probability are only approximations of the "true" solution. The other source of difficulties in GA is the estimation of the solution errors. Gener- ally, in order to obtain the resulting uncertainties the stochastic techniques, e.g. "quick and dirty" Monte Carlo, are used [Press et al., 1992, pp. 689-699].

Charbonneau [1995] presents the examples of solving optimization problems by means of the GA as well as provides an open source optimization code PIKAI which maximizes a function $f\left(x_{1}, x_{2}, x_{3}, \ldots, x_{n}\right)$ in the $\mathrm{n}$-dimensional parameter space. PIKAI has the following major controlling parameters which determine the performance of the algorithm: number of generations $N_{g}$ (or number of iterations), size of the populations $N_{p}$ in each generation, mutation rate $p_{m}$ and crossover rate $p_{c} . N_{g}$ and $N_{p}$ are most critical parameters which determine the performance of the algorithm. The iterative GA can be terminated either after a given number of iterations or when some convergence criteria are satisfied, e. g. improvement of the best solutions (the fitness level of the best individual) falls below a given threshold. In our case the size of the populations was $N_{p}=100$ and GA was terminated after $N_{g}=300$ iterations. These numbers have been chosen as a reasonable compromise between computation time and accuracy of $\chi^{2}$ minimization.

\section{Results and error analysis}

As was mentioned in the previous section the GA can operate in the whole space of the free parameters of the model and does not require the initial guess of the parameters. Nevertheless, in order to reduce the computing time, we limited the space of possible parameters in the following way: 1) tilt angles of each effective antenna vector $\vec{h}$ relative to physical antenna roods (see tables 1, 2, 3 columns "Mechanical" ) are limited to be $30^{\circ}$, and 2 ) the effective length of each antenna $|\vec{h}|$ does not exceed $\pm 50 \%$ offset from the length of the short dipole antenna $(L / 2)$.

The red lines in Figure 2 show the model predicted values for DF2 mode fitted to the AKR observations using GA. As seen, the model fit the observations well. The resulting effective antenna lengths for different antenna configuration (optimized model parameters) are listed in tables 1,2 and 3.

One important point is that since measurements are contaminated by instrumental systematic errors as well as a random noise the best fitted set of parameters is not a unique realization of the "true" parameters. Therefore, the errors of the fitted parameters have to be carefully estimated. In a nonlinear 
STEREO-B/WAVES, DF2 mode, 01/31/2007 04:30 - 15:00 UT
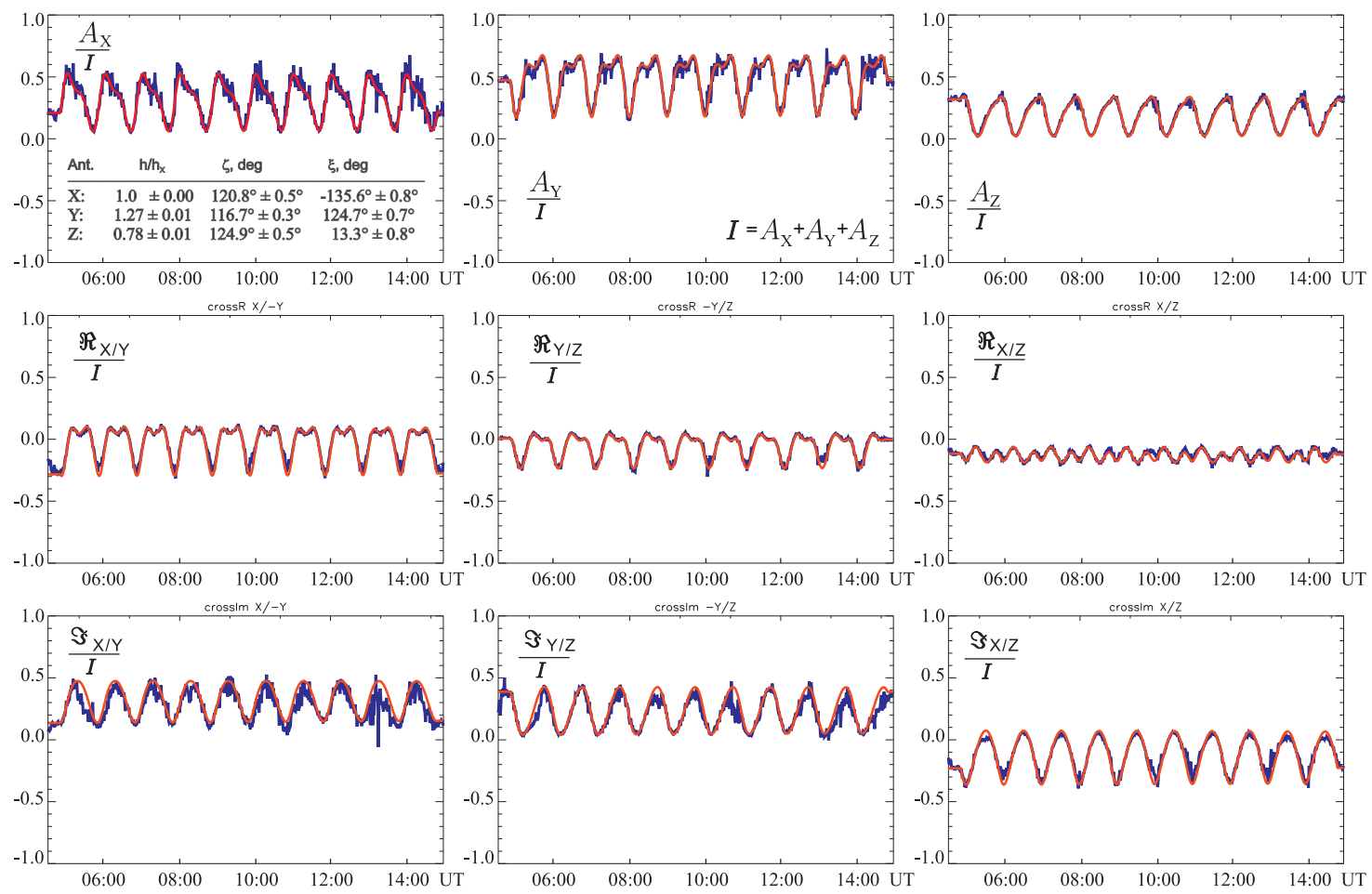

Figure 2. Modeled analytic signals (red lines) fitted to the measured output voltages at each channel of the STEREO-B HFR1 receiver working in Direction Finding (DF2) mode (blue lines). The modeled and measured time profiles are normalized by $I=A_{X}+A_{Y}+A_{Z}$. In DF2 mode all three antennas $(\mathrm{X}, \mathrm{Y}, \mathrm{Z})$ are operated. The best fitted parameters which define the effective antenna vectors are tabulated.

least-squares methods (e.g. conjugate gradient-type techniques) the errors can be obtained from inverse Hessian matrix of second derivatives evaluated at the best solution. Since GA does not use the derivatives the best way is to use a Monte Carlo simulation in which a large number of synthetic data sets are generated from the best fitted model, adding random noise in accordance with the measurement errors. The GA algorithm is applied for each of these sets and finally, the standard errors of the obtained estimators are determined.

In order to estimate the fit errors, we used a so called "Quick and dirty" Monte Carlo or Bootstrap method (e.g. Efron and Tibshirani [1993]) - a powerful technique which can be applied even without information about a true underlying error distribution of the observations. The bootstrap method generates the synthetic data sets by randomly resampling with replacing of approx $37 \%$ of the original data (see for more details [Press et al., 1992, pp. 689-699]). We synthesized $\mathrm{M}=500$ bootstrapped data sets using the measured temporal variations of the AKR intensity and then applied the fitting procedure based on GA to each of these synthetic data sets (each synthetic set has the same number of points as the measured AKR intensity profile). It provides 500 sets of parameters $\mathbf{X}_{\mathbf{1}}, \ldots, \mathbf{X}_{\mathbf{m}}$ each of which define the effective antenna length vectors. Figure 3 shows the histogram of the bootstrapped parameters. Then, since the distribution of the bootstrapped parameters $\mathbf{X}_{\mathbf{i}}$ is approximately normal (as seen in Figure 3), the widths of the distributions give us the standard deviations $\sigma$ of the estimated parameters.

The same calculations have been performed also for the SWAVES antennas working in DF1 mode and the final results with one sigma errors are listed in tables 1, 2 and 3.

\section{Discussion and summary}



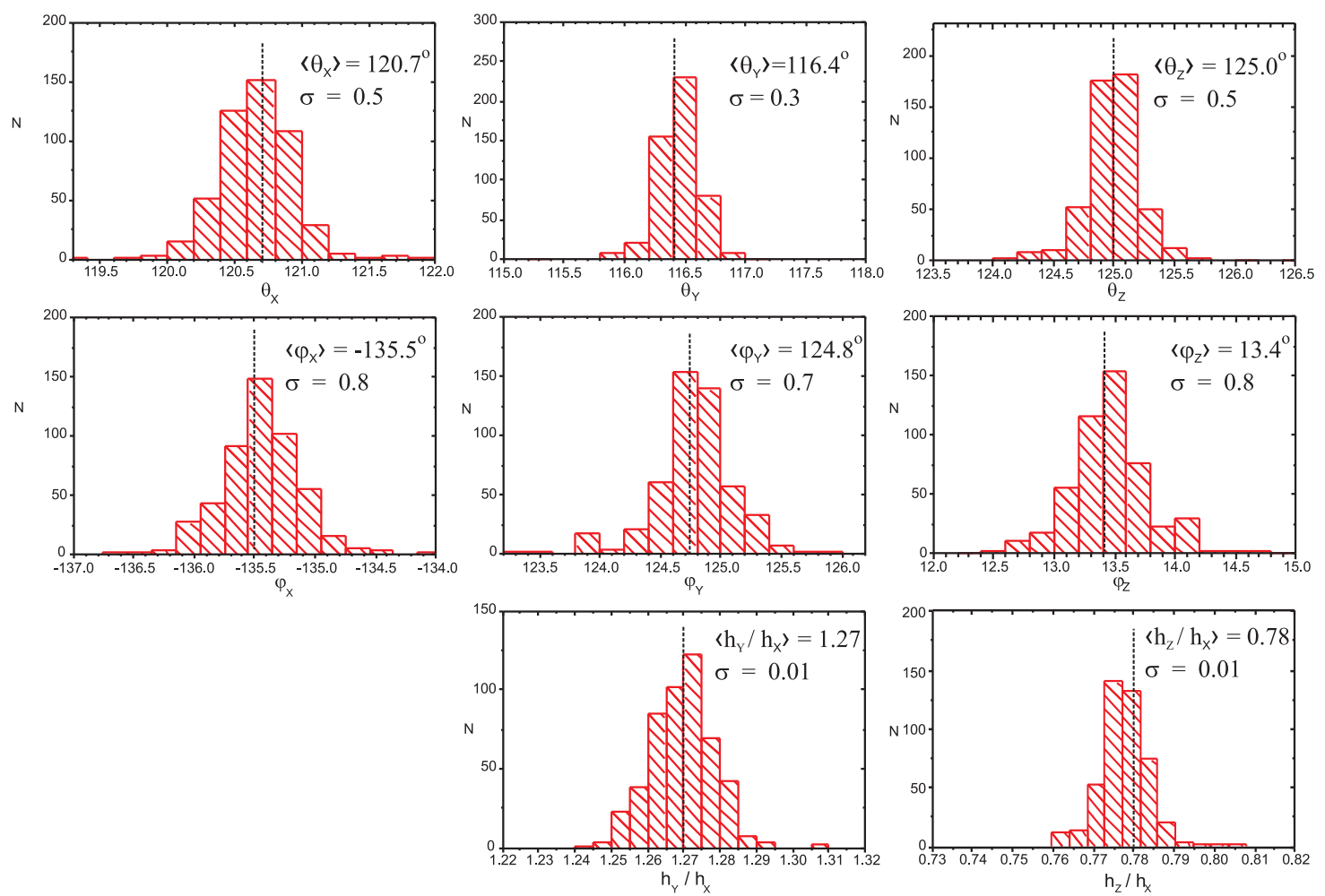

Figure 3. Bootstrap error analysis of best fitted parameters $\mathbf{X}=$ $\left\{h_{X}, \zeta_{X}, \xi_{X} ; h_{Y} / h_{X}, \zeta_{Y}, \xi_{Y} ; h_{Z} / h_{X}, \zeta_{Z}, \xi_{Z}\right\}$ which define the effective vectors of the SWAVES antennas for DF2 mode. The histograms show the distributions of the fitted parameters obtained by application of the genetic optimization algorithm to $M=500$ synthetic data sets generated by means of the bootstrap method. The standard deviations of the $\mathbf{X}$ elements are the same as the standard deviations $\sigma$ of the bootstrap histograms. More details are given in the penultimate paragraph of the section 4 .

Table 1. Results of in-flight calibration of the SWAVES antennas in direction finding mode DF2 (monopoles $X, Y$, and $Z$ ). The column headed "Mechanical" contains relative lengths and directions of the antenna rods. The column headed "Effective" contains the components of the effective antenna lengths and directions with one sigma errors. $\zeta$ and $\xi$ are defined in the STEREO/WAVES spherical coordinates, described in section 3.2, and $\theta$ and $\varphi$ are colatitude and azimuths of the antenna vectors in the spherical coordinates related to the spacecraft main axis, i.e. $\theta$ is measured from $\mathrm{Z}$ spacecraft axis, and $\varphi$ is azimuths angle from $\mathrm{X}$ spacecraft axis.

\begin{tabular}{cccccccc}
\hline & \multicolumn{4}{c}{ Mechanical } & & \multicolumn{3}{c}{ Effective } \\
\cline { 2 - 4 } \cline { 6 - 8 } Ant. & $h / h_{X}$ & $\zeta$ & $\xi$ & & $h / h_{X}$ & $\zeta$ & $\xi$ \\
\hline$X$ & 1.0 & $125.26^{\circ}$ & $-120.0^{\circ}$ & & 1.00 & $120.8^{\circ} \pm 0.5^{\circ}$ & $-135.6^{\circ} \pm 0.8^{\circ}$ \\
$Y$ & 1.0 & $125.26^{\circ}$ & $120.0^{\circ}$ & & $1.27 \pm 0.01$ & $116.7^{\circ} \pm 0.3^{\circ}$ & $124.7^{\circ} \pm 0.7^{\circ}$ \\
$Z$ & 1.0 & $125.26^{\circ}$ & $0.0^{\circ}$ & & $0.78 \pm 0.01$ & $124.9^{\circ} \pm 0.5^{\circ}$ & $13.3^{\circ} \pm 0.8^{\circ}$ \\
\hline Ant. & $h / h_{X}$ & $\theta$ & $\varphi$ & & $h / h_{X}$ & $\theta$ & $\varphi$ \\
\hline$X$ & 1.0 & $65.9^{\circ}$ & $230.8^{\circ}$ & & 1.00 & $52.1^{\circ} \pm 0.7^{\circ}$ & $229.6^{\circ} \pm 0.6^{\circ}$ \\
$Y$ & 1.0 & $65.9^{\circ}$ & $129.2^{\circ}$ & & $1.27 \pm 0.01$ & $59.4^{\circ} \pm 0.6^{\circ}$ & $121.5^{\circ} \pm 0.4^{\circ}$ \\
$Z$ & 1.0 & $144.7^{\circ}$ & $180.0^{\circ}$ & & $0.78 \pm 0.01$ & $143.0^{\circ} \pm 0.5^{\circ}$ & $161.8^{\circ} \pm 1.0^{\circ}$ \\
\hline
\end{tabular}

The final set of antenna parameters of STEREO/ WAVES obtained by the in-flight calibration (for direction finding mode DF2) are compared with rheometry and computer simulations previously performed in Graz and reported in Macher et al. [2007] and Oswald et al. [2009]. Table 4 summarizes the elements of the antenna effective length vectors using the different methods. For each method we have also checked the goodness of the fit by calculating 
Table 2. Results of in-flight calibration of the SWAVES antennas in mode DF1 "13" (monopole $X-\operatorname{dipole} Z Y$ ).

\begin{tabular}{|c|c|c|c|c|c|c|}
\hline \multirow[b]{2}{*}{ Ant. } & & \multicolumn{2}{|c|}{ Mechanical ('13' mode) } & \multicolumn{3}{|c|}{ Effective ('13' mode) } \\
\hline & $h / h_{X}$ & $\bar{\zeta}$ & $\bar{\xi}$ & $h / h_{X}$ & $\bar{\zeta}$ & $\bar{\xi}$ \\
\hline $\bar{X}$ & 1.0 & $125.26^{\circ}$ & $-120.0^{\circ}$ & 1.00 & $120.4^{\circ} \pm 0.8^{\circ}$ & $-136.3^{\circ} \pm 1.1^{\circ}$ \\
\hline$Z Y$ & 1.414 & $90.0^{\circ}$ & $150.0^{\circ}$ & $1.32 \pm 0.01$ & $92.4^{\circ} \pm 1.0^{\circ}$ & $146.7^{\circ} \pm 1.0^{\circ}$ \\
\hline Ant. & $h / h_{X}$ & $\theta$ & $\varphi$ & $h / h_{X}$ & $\theta$ & \\
\hline$X$ & 1.0 & $65.9^{\circ}$ & $230.8^{\circ}$ & 1.00 & $51.4^{\circ} \pm 0.9^{\circ}$ & $229.7^{\circ} \pm 0.9^{\circ}$ \\
\hline$Z Y$ & 1.414 & $30.0^{\circ}$ & $90.0^{\circ}$ & $1.32 \pm 0.01$ & $33.4^{\circ} \pm 1.0^{\circ}$ & $94.4^{\circ} \pm 1.8^{\circ}$ \\
\hline
\end{tabular}

Table 3. Results of in-flight calibration of the SWAVES antennas in dipole mode DF1 "31" ( monopole $Z-$ dipole $X Y$ ).

\begin{tabular}{|c|c|c|c|c|c|c|}
\hline \multirow[b]{2}{*}{ Ant. } & \multicolumn{3}{|c|}{ Mechanical ('31' mode) } & \multicolumn{3}{|c|}{ Effective ('31' mode) } \\
\hline & $h / h_{Z}$ & $\zeta$ & $\xi$ & $h / h_{Z}$ & $\zeta$ & $\xi$ \\
\hline $\begin{array}{c}X Y \\
Z\end{array}$ & $\begin{array}{c}1.414 \\
1.0\end{array}$ & $\begin{array}{c}90.0^{\circ} \\
125.26^{\circ}\end{array}$ & $\begin{array}{c}90.0^{\circ} \\
0.0^{\circ}\end{array}$ & $\begin{array}{c}1.76 \pm 0.01 \\
1.00\end{array}$ & $\begin{array}{c}90.6^{\circ} \pm 0.7^{\circ} \\
124.3^{\circ} \pm 0.7^{\circ}\end{array}$ & $\begin{array}{l}88.7^{\circ} \pm 0.8^{\circ} \\
13.8^{\circ} \pm 1.0^{\circ}\end{array}$ \\
\hline $\begin{array}{l}\text { Ant. } \\
X Y \\
Z Y\end{array}$ & $\begin{array}{c}h / h_{X} \\
1.0 \\
1.414\end{array}$ & $\begin{array}{c}\theta \\
90.0^{\circ} \\
144.7^{\circ}\end{array}$ & $\begin{array}{c}\varphi \\
90.0^{\circ} \\
180.0^{\circ}\end{array}$ & $\begin{array}{c}h / h_{X} \\
1.00 \\
1.33 \pm 0.01\end{array}$ & $\begin{array}{c}\theta \\
91.3^{\circ} \pm 0.8^{\circ} \\
143.3^{\circ} \pm 0.7^{\circ}\end{array}$ & $\begin{array}{c}\varphi \\
90.6^{\circ} \pm 0.7^{\circ} \\
160.7^{\circ} \pm 1.3^{\circ}\end{array}$ \\
\hline
\end{tabular}

the reduced chi-squared: $\xi=\chi^{2} /(N * M-\nu-1)$, where $\chi^{2}$ is defined by equation $(3), N$ is the total number of observation sets, $M$ is the number of output channels, and $\nu$ the number of fitted parameters of the model. $\xi$ describes the discrepancy between the observed signals of the AKR and the model predicted values, as calculated by equation (3) with the effective antenna length vectors obtained by the respective method. As can be seen from the table, the smallest $\xi$ corresponds to the in-flight results, although the values of the goodness of the fit $(\xi)$ are very similar for all methods.

Also, the results of the experimental rheometry and numerical computer simulations well agree with the in-flight calibrations analysis within the inherent inaccuracy of the methods. In particular, the angular components of the antenna effective length vectors obtained by the different methods are within $3 \sigma$ errors of each other. This confirms the high reliability of the rheometry and computer modeling for the calibration of the antenna system. For the effective lengths we find very small but significant differences between the present results and those obtained by former authors (Macher et al. [2007]; Oswald et al. [2009]; Zaslavsky et al. [2011]). The most plausible explanation is that the base capacitances associated with the antennas $X, Y$ and $Z$ are not exactly the same. Since base capacitances shorten the effective length of an antenna, the results given in Table 4 indicate $C_{Y} / C_{X}<1$ and $C_{Z} / C_{X}>1$ (the latter being confirmed by Zaslavsky et al. [2011], who have determined $h_{X}$ and $h_{Z}$, but not $h_{Y}$ ).

As was mentioned in section 3.1 only STEREO-B observed the AKR during the roll maneuvers and, therefore, the in-flight calibration was applied only for the SWAVES antenna system onboard STEREOB. Nevertheless, since the two STEREO spacecraft are almost identical we expect that the antenna effective length vectors of the WAVES experiment on STEREO-A are very close to STEREO-B, determined by the in-flight calibration. This assumption was confirmed by rheometry and computer simulations. However, Zaslavsky et al. [2011] found slightly different results for STEREO-A and STEREO-B by analysis of the effective antenna lengths using the galactic background.

The proper calibration of the reception properties of the spaceborne antenna system is one of the major data processing tasks yielding an accurate evaluation of the radio data, which is very important especially for the direction finding of electromagnetic waves. The in-flight calibration approach, presented in this paper, gives us reliable results because this method uses the data acquired by the "real" antenna-receiver configuration which are difficult to estimate or measure during the spacecraft ground-based calibration procedures. For example, the accurate determination of the stray and base capacitances of the antennas plays an important role in achieving realistic results by rheometry and computer simulation methods [Macher et al., 2007]. 
Table 4. Results of the STEREO-B/WAVES antenna calibration by in-flight calibration, computer simulation, and rheometry (computer simulation with CONCEPT and for "loaded" feeds and rheometry results from Oswald et al. [2009]). Column "Mechanical" contains the values which describe the relative length and direction of the antenna rods. The values in the parentheses denote the standard deviation of the obtained values. $\xi$ is the reduced chi-squared statistic which is to check the goodness of the fit.

\begin{tabular}{|c|c|c|c|c|c|}
\hline Ant & & In-flight & Computer simulation & Rheometry & Mechanica \\
\hline \multirow{3}{*}{$X$} & $\overline{h_{X} / h_{X}}$ & 1.00 & 1.00 & 1.00 & 1.00 \\
\hline & $\zeta$, deg & $120.8^{\circ}(0.5)$ & $119.9^{\circ}$ & $121.3^{\circ}$ & $125.3^{\circ}$ \\
\hline & $\xi, d e g$ & $-135.6^{\circ}(0.8)$ & $-135.3^{\circ}$ & $-135.4^{\circ}$ & $-120.0^{\circ}$ \\
\hline \multirow{3}{*}{$Y$} & $h_{Y} / h_{X}$ & $1.27(0.01)$ & 1.21 & 1.26 & 1.00 \\
\hline & $\zeta, \mathrm{deg}$ & $116.7^{\circ}(0.3)$ & $114.4^{\circ}$ & $115.1^{\circ}$ & $125.3^{\circ}$ \\
\hline & $\xi$, deg & $124.7^{\circ}(0.7)$ & $127.3^{\circ}$ & $126.8^{\circ}$ & $120.0^{\circ}$ \\
\hline \multirow{3}{*}{$Z$} & $h_{Z} / h_{X}$ & $0.78(0.01)$ & 0.81 & 0.84 & 1.00 \\
\hline & $\zeta, \mathrm{deg}$ & $124.9^{\circ}(0.5)$ & $124.7^{\circ}$ & $125.3^{\circ}$ & $125.3^{\circ}$ \\
\hline & $\xi$, deg & $13.3^{\circ}(0.8)$ & $15.5^{\circ}$ & $16.4^{\circ}$ & $0.0^{\circ}$ \\
\hline$\xi$ & & 1.08 & 1.26 & 1.25 & 11.3 \\
\hline
\end{tabular}

Since the AKR is observed in the frequency range below $1 \mathrm{MHz}$, we can only study the reception properties of the antenna in electrically short dipole approximation when the radio wavelength is much greater than the size of the antenna system - i.e. $L_{\text {antenna }} \ll \lambda_{\text {wave }}$. In this frequency range (also called quasi-static frequency range) the effective length vectors are real and constant (independent of direction). The effective antenna length vectors on higher frequencies will be significantly different, especially for frequencies close to the antenna resonances [Oswald et al., 2009].

\section{Acknowledgments.}

The authors are pleased to acknowledge the Plasma Physics Data Center (CDPP) team for providing the STEREO/WAVES data. This work was financed by the Austrian Science Fund (FWF projects P23762-N16 and P20680-N16). In France the S/WAVES instrument has been developed with the support of both CNES and CNRS.

\section{References}

Bougeret, J. L., et al., S/WAVES: The Radio and Plasma Wave Investigation on the STEREO Mission, Space Science Reviews, 9, doi:10.1007/s11214-007-9298-8, 2008.

Cecconi, B., and P. Zarka, Direction finding and antenna calibration through analytical inversion of radio measurements performed using a system of two or three electric dipole antennas on a three-axis stabilized spacecraft, Radio Science, 40, 3003, doi: 10.1029/2004RS003070, 2005.

Charbonneau, P., Genetic Algorithms in Astronomy and Astrophysics, Astrophys. J. (Supp.), 101, 309, doi: 10.1086/192242, 1995.

Efron, B., and R. J. Tibshirani, An Introduction to the Bootstrap, Chapman \& Hall, New York, 1993.
Fainberg, J., S. Hoang, and R. Manning, Measurements of Distributed Polarized Radio Sources from Spinning Spacecraft - Effect of a Tilted Axial Antenna - ISEE3 Application and Results, Astron. Astrophys., 153, $145,1985$.

Fischer, G., W. Macher, H. O. Rucker, H. P. Ladreiter, D. F. Vogl, and Cassini/Rpws Team, Wire-grid Modeling of Cassini Spacecraft for the Determination of Effective Antenna Length Vectors of the RPWS Antennas, in Planetary Radio Emissions V, edited by H. O. Rucker, M. L. Kaiser, and Y. Leblanc, 347, Austrian Academy of Sciences Press, Veinna, 2001.

Gurnett, D. A., The earth as a radio source: Terrestrial kilometric radiation, J. Geophys. Res., 79, 4227, 1974.

Hanasz, J., M. Panchenko, H. de Feraudy, R. Schreiber, and M. M. Mogilevsky, Occurrence distributions of the auroral kilometric radiation ordinary and extraordinary wave modes, J. Geophys. Res. (Space Physics), 108(A11), doi:10.1029/2002JA009579, 2003.

Holland, J. H., Adaptation in natural and artificial systems: an introductory analysis with applications to biology, control and artificial intelligence, Ann Arbor: University of Michigan Press, 1975.

Ladreiter, H. P., P. Zarka, A. Lecacheux, W. Macher, H. O. Rucker, R. Manning, D. A. Gurnett, and W. S. Kurth, Analysis of electromagnetic wave direction finding performed by spaceborne antennas using singular-value decomposition techniques, Radio Science, 30, 1699, 1995.

Lecacheux, A., Direction Finding and Polarization Measurements of SKR, in Planetary Radio Emissions VII, edited by Rucker, H. O., Kurth, W. S., Louarn, P., Fischer, G., 13, 2011.

Lecacheux, A., and A. Ortega-Molina, Polarization and localization of the Uranian radio sources, J. Geophys. Res., 92, 15,148, 1987.

Macher, W., Transfer matrix description of multiport antennas and its application to the Mars Express/MARSIS radar, Ph.D. thesis, Graz University of Technology, Austria, 2008.

Macher, W., and T. H. Oswald, Radius correction formula for capacitances and effective length vectors of monopole and dipole antenna systems, Radio Science, 46, RS1011, doi:10.1029/2010RS004446, 2011. 
Macher, W., T. H. Oswald, G. Fischer, and H. O. Rucker, Rheometry of multi-port spaceborne antennas including mutual antenna capacitances and application to STEREO/WAVES, Measurement Science and Technology, 18, 3731, doi:10.1088/0957-0233/18/12/008, 2007.

Mera, N. S., L. Elliott, and D. B. Ingham, A multipopulation genetic algorithm approach for solving illposed problems, Computational Mechanics, 33, 254, 10.1007/s00466-003-0526-0, 2004.

Mutel, R. L., D. A. Gurnett, and I. W. Christopher, Spatial and Temporal Properties of AKR Burst Emission Derived from Cluster WBD VLBI Studies, Annales Geophysicae, 22, 2625, 2004.

Oswald, T. H., W. Macher, H. O. Rucker, G. Fischer, U. Taubenschuss, J. L. Bougeret, A. Lecacheux, M. L. Kaiser, and K. Goetz, Various methods of calibration of the STEREO/WAVES antennas, Advances in Space Research, 43, 355, doi:10.1016/j.asr.2008.07.017, 2009.

Panchenko, M., Polarimetry of auroral kilometric radiation with a triaxial nonorthogonal antenna system, $R a$ dio Science, 39(RS6010), doi:10.1029/2004RS003039, 2004.

Panchenko, M., J. Hanasz, and H. O. Rucker, Estimation of linear wave polarization of the auroral kilometric radiation, Radio Science, 43, 1006, doi: 10.1029/2006RS003606, 2008.

Panchenko, M., et al., Daily variations of auroral kilometric radiation observed by STEREO, Geophys. Res. Lett., 36, L06102, doi:10.1029/2008GL037042, 2009.

Press, W., S. Teukolski, W. Vetterling, and B. Flannery (Eds.), Numerical Recipes in C: The Art of Scientific Computing, 2nd ed., Cambridge University Press, 1992.

Riddle, A. C., Antenna pattern testing on spacecraft model, Rep. MEM-MJS-76-S100, pp. Lab. for Extraterr. Phys., NASA Goddard Space Flight Center, Greenbelt, Md., 1976.

Rucker, H. O., W. Macher, R. Manning, and H. P. Ladreiter, Cassini model rheometry, Radio Science, 31, $1299,1996$.

Rucker, H. O., M. Sampl, M. Panchenko, T. Oswal, D. Plettemeier, M. Maksimovic, and W. Macher, Implications of Antenna System Calibration on Spacecraft Design and Radio Data Analysis, Planetary, Solar and Heliospheric Radio Emissions (PRE VII), 475, Austrian Academy of Sciences Press, Veinna, 2011.
Sampl, M., H. O. Rucker, T. H. Oswald, D. Plettemeier, M. Maksimovic, and W. Macher, Numerical Simulations of the Solar Orbiter Antenna System RPW ANT, Planetary, Solar and Heliospheric Radio Emissions (PRE VII), 487, 2011.

Sampl, M., W. Macher, C. Gruber, T. Oswald, H. O. Rucker, and M. Mogilevsky, Calibration of Electric Field Sensors Onboard the Resonance Satellite, IEEE Transactions on Antennas and Propagation, 60, 267, doi:10.1109/TAP.2011.2167918, 2012.

Taubenschuss, U., The linear prediction theory applied to cassini data, M.Sc. thesis, Karl Franzens University of Graz, Austria, 2005.

Vogl, D. F., et al., In-flight calibration of the CassiniRadio and Plasma Wave Science (RPWS) antenna system for direction-finding and polarization measurements, J. Geophys. Res., 109, A09S17, doi: 10.1029/2003JA010261, 2004.

Wang, L., and T. D. Carr, Recalibration of the Voyager PRA antenna for polarization sense measurement, $A s$ tron. Astrophys., 281, 945, 1994.

Zarka, P., B. Cecconi, and W. S. Kurth, Jupiter's lowfrequency radio spectrum from Cassini/Radio and Plasma Wave Science (RPWS) absolute flux density measurements, J. Geophys. Res. (Space Physics), 109, A09S15, doi:10.1029/2003JA010260, 2004.

Zaslavsky, A., N. Meyer-Vernet, S. Hoang, M. Maksimovic, and S. D. Bale, On the antenna calibration of space radio instruments using the galactic background: General formulas and application to STEREO/WAVES, Radio Science, 46, RS2008, doi: 10.1029/2010RS004464, 2011.

M. Panchenko, W. Macher, H.O. Rucker, G. Fischer, T. Oswald Space Research Institute Austrian Academy of Sciences, Schmiedlstrasse 6, A-8042 Graz, Austria (mykhaylo.panchenko@oeaw.ac.at)

B. Cecconi, M. Maksimovic LESIA, Observatoire de Paris, 5, place Jules Janssen, 92195 MEUDON Cedex, France.

(Received _.) 